\title{
Maxillofacial surgeon secures grant to develop virtual reality surgical training
}

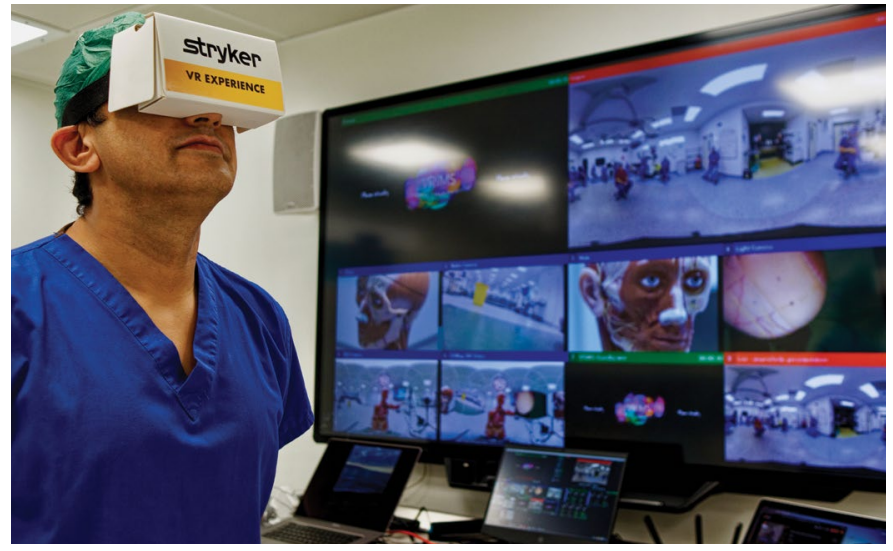

Professor Jag Dhanda, a Consultant Maxillofacial/Head and Neck Surgeon (OMFS) at the Queen Victoria Hospital, East Grinstead and Honorary Professor of Surgery at the Brighton and Sussex Medical School, has won a $£ 1$ million grant from Health Education England (HEE) to develop virtual reality (VR) and augmented reality (AR) surgical training resources.

Professor Dhanda (pictured) said: 'I hope I can demonstrate how this exciting new immersive technology can overcome the dramatic impact that COVID has had on medical and surgical education. I want to prove that $\mathrm{VR}$ and $\mathrm{AR}$ can deliver lasting improvements to surgical training across a wide range of skills, and validate their use as medical education resources'

He said that the General Medical Council had found that the COVID pandemic had had a significant impact on surgical training: 'Trainees say they haven't been able to compensate for the lost training opportunities, and fear they have not progressed curriculum competencies. So, I believe this emerging technology is an addition to conventional face-to-face training - and has the potential to replace it!'

Professor Dhanda is the founder and clinical lead of Virtual Reality in Medicine and Surgery (VriMS), a free-for-trainee platform that live-streams surgical training videos in virtual reality.

Professor Dhanda added: 'A course has just finished where new content was live-streamed and previous content restreamed. And, there will be an additional hands-on course for OMFS surgeons (CRANIOMAX), ENT and neurosurgeons that will also feature augmented reality applications for endonasal skull base access and procedures.

With the help of Professor Claire Smith, Head of Anatomy at Brighton and Sussex Medical School, cadaver demonstrations of surgical techniques using virtual reality have been live-streamed over five one-week courses since the start of the pandemic.

Professor Dhanda said he also wanted VriMS to use eye tracking and physiological sensors with haptics to show adaptions to training and trainee responses to VR/AR technology. 'I plan to use the grant to develop a true VR basic life support app for health care professionals and teachers, and augmented reality apps for mandatory training with manual handling. Also, true VR apps for basic surgical skills such as suturing and local skin flaps'.

Professor Dhanda now plans a major project grant application to Innovate UK, part of UK Research and Innovation, a non-departmental public body funded by a grant-in-aid from the UK government. 